ARTICLE

\title{
Metal oxide charge transfer complex for effective energy band tailoring in multilayer optoelectronics
}

Moohyun Kim¹,4, Byoung-Hwa Kwon (i) 2,4, Chul Woong Joo², Myeong Seon Cho, Hanhwi Jang ${ }^{1}$, Ye ji Kim', Hyunjin Cho ${ }^{1}$, Duk Young Jeon ${ }^{1}$, Eugene N. Cho ${ }^{3 凶} \&$ Yeon Sik Jung (1) ${ }^{1 凶}$

Metal oxides are intensively used for multilayered optoelectronic devices such as organic light-emitting diodes (OLEDs). Many approaches have been explored to improve device performance by engineering electrical properties. However, conventional methods cannot enable both energy level manipulation and conductivity enhancement for achieving optimum energy band configurations. Here, we introduce a metal oxide charge transfer complex ( $\mathrm{NiO}: \mathrm{MoO}_{3}$-complex), which is composed of few-nm-size $\mathrm{MoO}_{3}$ domains embedded in $\mathrm{NiO}$ matrices, as a highly tunable carrier injection material. Charge transfer at the finely dispersed interfaces of $\mathrm{NiO}$ and $\mathrm{MoO}_{3}$ throughout the entire film enables effective energy level modulation over a wide work function range of $4.47-6.34 \mathrm{eV}$ along with enhanced electrical conductivity. The high performance of $\mathrm{NiO}: \mathrm{MoO}_{3}$-complex is confirmed by achieving $189 \%$ improved current efficiency compared to that of $\mathrm{MoO}_{3}$-based green OLEDs and also an external quantum efficiency of $17 \%$ when applied to blue OLEDs, which is superior to 1,4,5,8,9,11-hexaazatriphenylene-hexacarbonitrile-based conventional devices.

\footnotetext{
${ }^{1}$ Department of Materials Science and Engineering, Korea Advanced Institute of Science and Technology (KAIST), 291 Daehak-ro, Yuseong-gu, Deajeon 34141, Republic of Korea. ${ }^{2}$ Reality Device Research Division, Electronics and Telecommunications Research Institute (ETRI), 218, Gajeong-ro, Yuseong-gu, Daejeon 34129, Republic of Korea. ${ }^{3}$ KAIST Institute for NanoCentury, Korea Advanced Institute of Science and Technology (KAIST), 291 Daehak-ro, Yuseong-gu, Daejeon 34141, Republic of Korea. ${ }^{4}$ These authors contributed equally: Moohyun Kim, Byoung-Hwa Kwon. ${ }^{\bowtie}$ email: nmecho@kaist.ac.kr; ysjung@kaist.ac.kr
} 
M etal oxide thin films are considered an attractive material for various functional applications because of their favorable energy band structure, excellent processability, and high stability ${ }^{1-3}$. In particular, this material system is intensively applied to multi-layered optoelectronic devices such as quantum-dot light-emitting diodes (QLEDs) and organic light-emitting diodes (OLEDs) for ensuring efficient charge transport and charge injection into the emission layer ${ }^{4-10}$. Conventional device design approaches usually adopt pristine metal oxides having compatible electrical properties with the target active layer ${ }^{11-14}$. However, with increasing variation of the active layer and demand for higher device performance, pristine metal oxide systems fall short of meeting the electrical property requirements to achieve both excellent charge transport and injection properties ${ }^{15-17}$.

The charge transport and injection properties are typically determined by two factors - the charge carrier density of each layer and the energy level configuration in a device. Although these two factors can be enhanced through engineering of material compositions or configurations, conventional techniques usually have fundamental limitations for tailoring both the charge transport and charge injection properties throughout the entire metal oxide layer. For example, the doping method, the representative conventional approach for improving the charge transport, provides insufficient modulation of the energy band structures of light-emitting devices ${ }^{10,18-20}$, and has a narrow tuning range with a doping limit of under 20 at. \% due to the formation of second phases and unwanted defects in the lattice ${ }^{19-21}$. On the other hand, surface charge transfer doping (SCTD), a recently emerged materials solution, can significantly modulate the energy level of metal oxides by forming acceptors or donors on the surface of the matrix material ${ }^{22-26}$. However, this enhancement effect is limited to a depth of $10 \mathrm{~nm}$ or less at the interface, and proved to be effective only in thin film transistors where the charge flows along the channel, while being unsuitable for multilayered optoelectronic applications where charge flows vertically to the region of the modified electronic structure $22-25$.

Herein, we propose a heterostructure based metal oxide charge transfer complex composed of two complementary metal oxides for modulating both the energy level and electrical conductivity by inducing effective charge transfer between the two metal oxides. This approach is aimed at widening the charge transfer range from the localized interface to the whole film by forming nanodomain dispersed fine heterostructure that constitutes a distinct energy band structure at the nanoscale. As an experimental verification, we selected nickel oxide $(\mathrm{NiO})$ as a p-type matrix and embedded molybdenum trioxide $\left(\mathrm{MoO}_{3}\right)$ nanoparticles (NPs) as strong p-type dopants with a high work function $^{27,28}$. The NiO: $\mathrm{MoO}_{3} \mathrm{NPs}$ heterostructure charge transfer complex ( $\mathrm{NiO}: \mathrm{MoO}_{3}$-complex) exhibits extensive controllability of the work function in a range of $4.47-6.34 \mathrm{eV}$ and electrical conductivity improvement of up to 2.4 times compared to that of pristine $\mathrm{NiO}$, without $\mathrm{MoO}_{3}$ concentration limit. The $\mathrm{NiO}: \mathrm{MoO}_{3}$ complex realizes $43 \%$ and $189 \%$ increased current efficiency in the green phosphorescent OLED system relative to those of pristine $\mathrm{NiO}$ and $\mathrm{MoO}_{3}$ by achieving a well-configured energy band structure for excellent electron-hole charge balance. Furthermore, $\mathrm{NiO}: \mathrm{MoO}_{3}$-complex is applied to an additional optoelectronic configuration of blue phosphorescent OLEDs, and their high capability and generality is verified on the basis of excellent performance $\left(32.6 \mathrm{~cd} \mathrm{~A}^{-1}\right.$ and $17 \%$ external quantum efficiency (EQE)), which is even higher than that of device using 1,4,5,8,9,11-hexaazatriphenylene-hexacarbonitrile (HATCN).

\section{Results}

Comparison and design of metal oxide charge transfer complex. Figure 1 compares carrier-density modulation methods (for metal oxides) - doping, surface charge transfer doping (SCTD), and formation of a heterostructure based metal oxide charge transfer complex (new approach in this work). The conventional doping method (Fig. 1a) enhances charge transport throughout the entire metal oxide film by incorporating dopants into the metal oxide lattice. Although the doping effect increases the charge transport ability throughout the entire film due to vacancy formation and charge compensation, the presence of the dopant does not provide sufficient energy level modulation to tune the charge injection property of the metal oxide. On the other hand, SCTD is able to modify the energy level, and as a result the charge injection property of the metal oxide film, by doping the surface of the matrix material prepared with various thin film deposition methods (Fig. 1b). Through interaction between the matrix material and the dopant layer, the nonidentical band position of the two materials promotes charge transfer, which, in turn, modifies the energy level at the interface of the two materials of the SCTD system. However, this phenomenon is localized to a $10 \mathrm{~nm}$ thick area at the interface of the bilayer, and therefore is unsuitable for multilayered optoelectronic applications where the charge flows vertically to the charge transfer region of the SCTD system.

The two enhancement methods mentioned above provide either full film property enhancement or energy level modulation, but not both. However, modulating the energy level and charge transport while providing the effect throughout the entire film is important for uniform and efficient charge injection and transport across the multilayer structure. Therefore, we designed a heterostructure charge transfer complex to realize charge transfer doping throughout the entire film by fabricating a matrix with embedded nanoparticles (NPs) (Fig. 1c). The heterostructure consisting of the matrix and the homogeneously distributed NPs forms nanodomains of distinct energy structures at the nanoscale that induce charge transfer at the interface between the two materials. In this regard, charge transfer interfaces can be generated throughout the entire layer, allowing the opportunity to modulate the energy level of the entire film, which cannot be achieved by conventional doping and SCTD. Furthermore, the energy level variation can be controlled by adjusting the NP ratio in the matrix to vary the amount of charge transfer interface, resulting in exact matching of the energy structure required to optimize the performance of the optoelectronic device.

Fabrication of NiO: $\mathrm{MoO}_{3}$ NPs charge transfer complex. We propose a charge transfer complex consisting of p-type $\mathrm{NiO}$ and n-type $\mathrm{MoO}_{3} \mathrm{NPs}$ as the donor and acceptor, respectively, to facilitate charge transfer for controlling the energy levels of the composite film. To verify the charge transfer effect between $\mathrm{NiO}$ and $\mathrm{MoO}_{3}$ through depth profile X-ray photoelectron spectroscopy (XPS) and ultraviolet photoelectron spectroscopy (UPS), we designed a $\mathrm{NiO}(20 \mathrm{~nm}) / \mathrm{MoO}_{3}(20 \mathrm{~nm})$ bilayer of a SCTD system (Supplementary Fig. 1). As seen from the energy diagram in Supplementary Fig. 1a, the work functions of $\mathrm{NiO}$ and $\mathrm{MoO}_{3}$ were estimated to be $\sim 4.5 \mathrm{eV}$ and $\sim 5.5 \mathrm{eV}$, respectively, which correspond to a sufficient energy level difference to promote charge transfer at the interface ${ }^{4,29}$. The shift in the work function around the $\mathrm{NiO} / \mathrm{MoO}_{3}$ interface with a width of about $10 \mathrm{~nm}$ (Supplementary Fig. 1c, d) provides evidence of charge transfer between $\mathrm{NiO}$ and $\mathrm{MoO}_{3}$.

Therefore, in order for $\mathrm{NiO}$ and $\mathrm{MoO}_{3}$ to function as a charge transfer complex, the $\mathrm{NiO}: \mathrm{MoO}_{3} \mathrm{NP}$ heterostructure of $\mathrm{NiO}$ :$\mathrm{MoO}_{3}$-complex should be constructed with separate $\mathrm{NiO}$ and $\mathrm{MoO}_{3}$ domains in the nanoscale without the formation of alloys, solid solutions, or voids. However, because the Ni-Mo-O system thermodynamically prefers the solid solution or alloy phases ${ }^{30}$, 


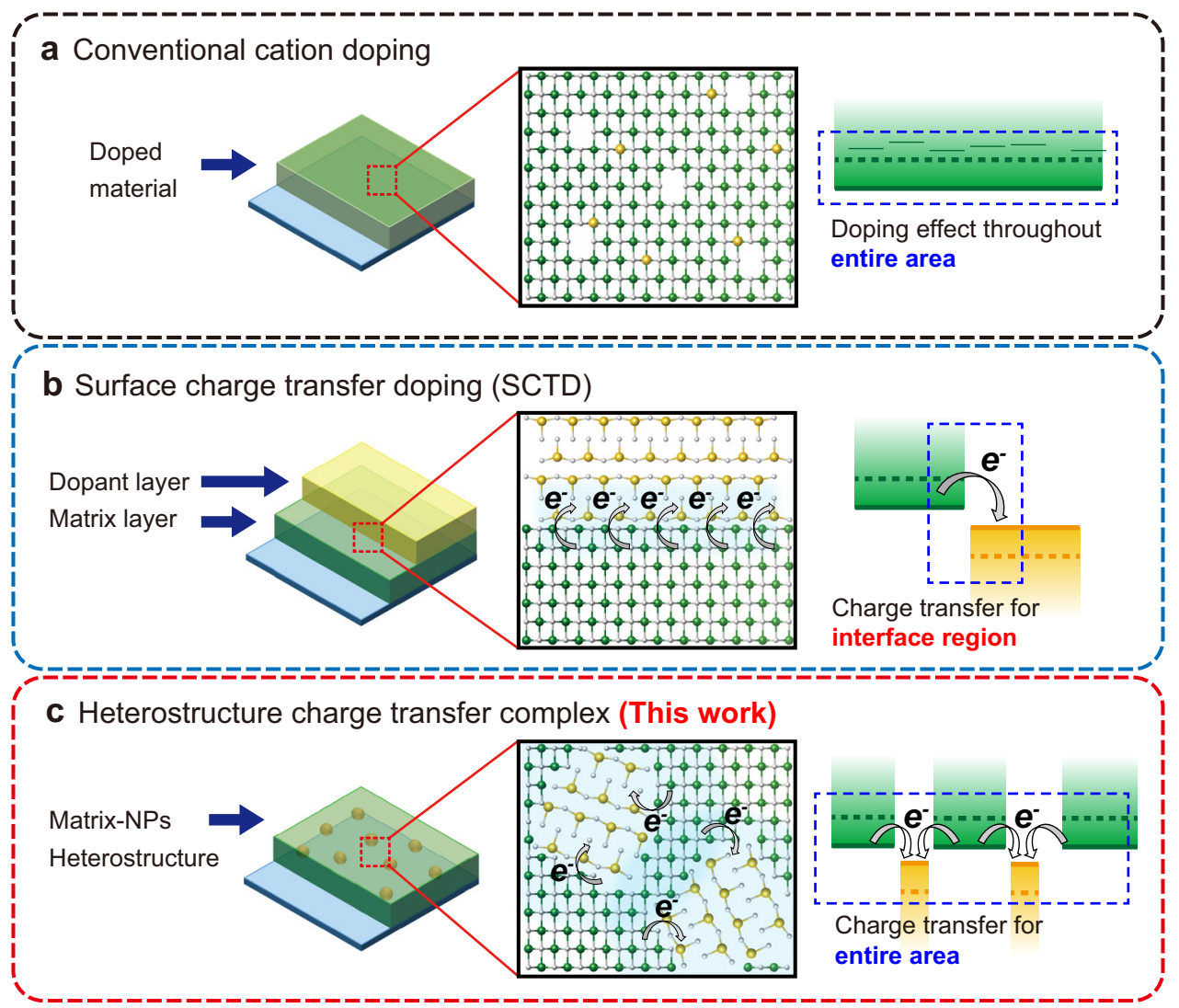

Fig. 1 Schematics of metal oxide material systems and energy level diagrams that compare conventional doping methods and our heterostructure charge transfer complex formation approach. a Conventional cation doping, b surface charge transfer doping (SCTD), and $\mathbf{c}$ heterostructure charge transfer complex formation (this work).

the formation of the proposed heterostructure is difficult with typical fabrication methods. Consequently, the heterostructure of the $\mathrm{NiO}$ and $\mathrm{MoO}_{3}$ NPs is thermodynamically metastable, and therefore careful design and understanding of the formation of the individual $\mathrm{NiO}$ and $\mathrm{MoO}_{3}$ domains is imperative. To form the heterostructure with distinct $\mathrm{NiO}$ and $\mathrm{MoO}_{3}$ domain, the $\mathrm{NiO}: \mathrm{MoO}_{3}$-complex was fabricated through a nanoparticle complexed sol-gel process. The sol-gel process was chosen because of the extensive controllability it provides over the processing stages (pyrolysis, condensation, and crystallization) in the low-temperature regime to form the wanted materials state ${ }^{31,32}$. To function as an embedded $\mathrm{MoO}_{3}$ domain inside the $\mathrm{NiO}$ matrix, the $\mathrm{MoO}_{3} \mathrm{NPs}(\sim 5 \mathrm{~nm}$ size) were synthesized beforehand using a microwave-assisted synthesis method (Supplementary Fig. 2) 33,34 . Surface roughness investigated by atomic force microscopy (AFM) of each pristine and complex films exhibit excellent smoothness and uniformity. The root mean square (RMS) roughness values of all the samples were less than $0.5 \mathrm{~nm}$, for example, an RMS roughness value of $0.17 \mathrm{~nm}$ for $\mathrm{NiO}: \mathrm{MoO}_{3} 50$ at. \% (Supplementary Fig. 3).

Systematic temperature XRD studies were performed on the sol-gel processed $\mathrm{NiO}, \mathrm{MoO}_{3} \mathrm{NPs}$, and $\mathrm{NiO}: \mathrm{MoO}_{3}-\mathrm{NP}$ complex to find the temperature window for forming the designed $\mathrm{NiO}: \mathrm{MoO}_{3}$-complex. XRD measurements of the sol-gel processed $\mathrm{NiO}$ (Fig. 2a) and $\mathrm{MoO}_{3}$ NPs (Fig. 2b) present patterns corresponding to $\mathrm{NiO}$ (JCPDS Card No.47-1049) and $\mathrm{MoO}_{3}$ (JCPDS Card No.05-0508), which were annealed at $250^{\circ} \mathrm{C}$ and $350{ }^{\circ} \mathrm{C}$, respectively. At $250^{\circ} \mathrm{C}$, the sol-gel processed $\mathrm{NiO}$ starts to undergo crystallization, while at $350^{\circ} \mathrm{C}$, the $\mathrm{MoO}_{3} \mathrm{NPs}$ undergo sintering and additional crystallization. The $\mathrm{NiO}: \mathrm{MoO}_{3}-\mathrm{NPs}$ complex (Fig. 2c) shows $\mathrm{NiO}$ peaks at $300{ }^{\circ} \mathrm{C}$ and $\mathrm{NiMoO}_{4}$ alloy
(JCPDS Card No.45-1042) peaks at $400^{\circ} \mathrm{C}$. These characterization results indicate that the sol-gel process remains in the pyrolysis and condensation step for insufficient heat conditions under $250{ }^{\circ} \mathrm{C}$ while the $\mathrm{NiO}$ phase and $\mathrm{NiMoO}_{4}$ alloy are formed at above $350^{\circ} \mathrm{C}$ (Supplementary Fig. 4). Therefore, we adopted a moderate annealing temperature of $300^{\circ} \mathrm{C}$ to promote only $\mathrm{NiO}$ crystallization in the complex while minimizing change in the $\mathrm{MoO}_{3} \mathrm{NPs}$ (Fig. 2d).

High-resolution transmission electron microscopy (HRTEM) was performed to characterize the NiO: $\mathrm{MoO}_{3}$ NPs heterostructures with varied $\mathrm{MoO}_{3} \mathrm{NP}$ compositions of $0 \%, 15$ at. \%, 30 at. $\%$, and 50 at. \%. The TEM images of the pristine $\mathrm{NiO}$ sample (Fig. 2e) exhibit $0.239 \mathrm{~nm}$ and $0.208 \mathrm{~nm}$ spacing distance (dspacing), which correspond to $\mathrm{NiO}$ (111) and $\mathrm{NiO}$ (200) planes, respectively ${ }^{35,36}$. The HRTEM images (Fig. $2 \mathrm{f}-\mathrm{h}$ ) demonstrate that $\mathrm{MoO}_{3} \mathrm{NPs}$ of $\sim 5 \mathrm{~nm}$ size (yellow dashed circle, $0.359 \mathrm{~nm}$ d-spacing of $\mathrm{MoO}_{3}$ (001) space $)^{37,38}$ are homogeneously incorporated in the $\mathrm{NiO}$ matrix with a linearly increasing proportion upon increasing the fraction of $\mathrm{MoO}_{3} \mathrm{NPs}$, confirming the formation of clearly distinct $\mathrm{NiO}$ and $\mathrm{MoO}_{3}$ domains. Fast Fourier transform (FFT) patterns of each HRTEM image further suggest the formation of separate $\mathrm{NiO}$ and $\mathrm{MoO}_{3}$ phases depending on the $\mathrm{MoO}_{3}$ NPs content. Another notable point from the TEM analysis is that $\mathrm{NiO}$ at the interface with $\mathrm{MoO}_{3}$ NPs is partially amorphized, which is supported by the broadening of the $\mathrm{NiO}$ peaks in the XRD patterns of the $\mathrm{NiO}: \mathrm{MoO}_{3}$ complex (Fig. $2 \mathrm{~h}$ and Supplementary Fig. 5). This phenomenon may contribute to the $\mathrm{Ni}^{2+}$ vacancy generation in the $\mathrm{NiO}$ lattice, which is directly related to the increase of the hole carrier concentration of $\mathrm{NiO}$. The XRD patterns of $\mathrm{MoO}_{3}$ did not appear in the measurements because of its extremely small size ${ }^{39}$. 

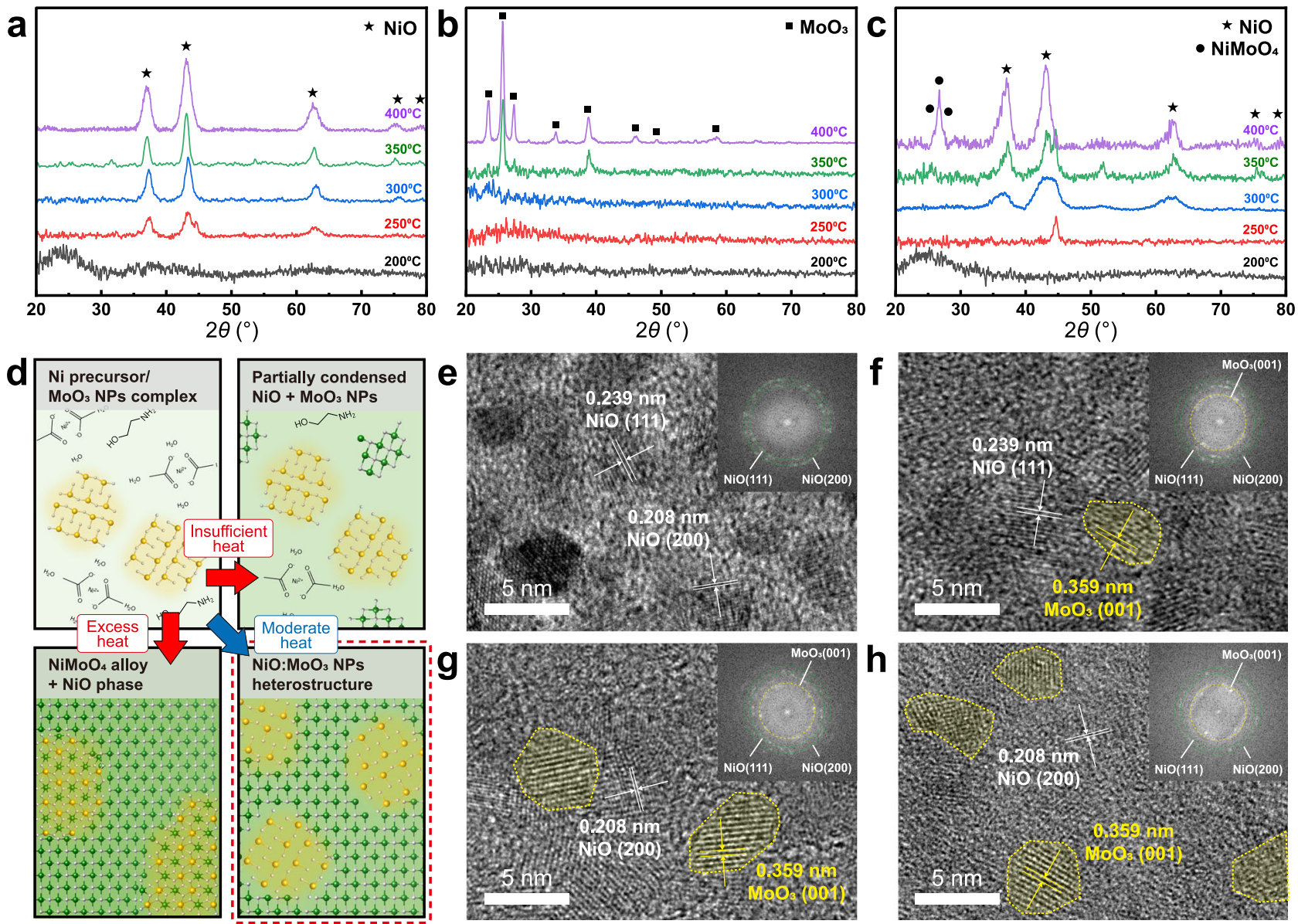

Fig. 2 Microstructure characterizations of $\mathbf{N i O}: \mathbf{M o O}_{3} \mathbf{~ N P s}$ heterostructure charge transfer complex. XRD patterns of a sol-gel processed NiO, $\mathbf{b} \mathrm{MoO}{ }_{3}$ $\mathrm{NPs}$, and $\mathbf{c} \mathrm{MoO}_{3} 20$ at.\% complexed NiO with various annealing temperatures. The symbols on the XRD peaks represent NiO (JCPDS Card No.47-1049), $\mathrm{MoO}_{3}$ (JCPDS Card No.05-0508), and $\mathrm{NiMoO}_{4}$ (JCPDS Card No.45-1042), respectively. d Schematics of the formation of the NiO:MoO$-\mathrm{M}_{3}-\mathrm{complex}$ depending on annealing conditions. High-resolution transmission electron microscopy (HRTEM) images of e NiO, f NiO: $\mathrm{MoO}_{3} 15$ at. \%, $\mathbf{g} \mathrm{NiO}: \mathrm{MoO}{ }_{3} \mathrm{NPs}$ 30 at. $\%$ and $\mathbf{h ~ N i O}: \mathrm{MoO}_{3} 50$ at. \% heterostructures (scale bar: $5 \mathrm{~nm}$ ). Insets of the HRTEM images correspond to fast Fourier transform (FFT) patterns.

Charge transfer in the NiO: $\mathrm{MoO}_{3}$-complex. To understand the charge transfer behavior at the interfaces between the two phases, an XPS analysis was conducted on the $\mathrm{NiO}: \mathrm{MoO}_{3}$-complex with varying compositions. As shown in Fig. $3 \mathrm{a}, \mathrm{Ni}^{3+}$ peaks shifted from $855.0 \mathrm{eV}$ to $856.0 \mathrm{eV}$ (dashed line, toward the higher binding energy) with an increasing fraction of $\mathrm{MoO}_{3} \mathrm{NPs}$ in the heterostructure. On the other hand, the Mo $3 d_{3 / 2}$ peak continuously shifted from $235.7 \mathrm{eV}$ to $235.1 \mathrm{eV}$ (to the lower binding energy) for composition change from $\mathrm{MoO}_{3}$ to $\mathrm{NiO}: \mathrm{MoO}_{3} 15$ at. \% (Fig. 3b, dashed line). These opposing shifts of Ni $2 p$ and Mo $3 d$ peaks as a function of $\mathrm{MoO}_{3}$ fraction are displayed quantitatively in Fig. 3c.

Peak shifts to the higher binding energies are due to loss of electrons, while peak shifts to lower binding energies are induced by gaining excess electrons. This phenomenon is attributed to the effective electron transfer (charge transfer) between the $\mathrm{NiO}$ and $\mathrm{MoO}_{3}$, as illustrated in Fig. 3d. Depending on the associated ratio of the two phases, the amount of peak shift representing the level of electron loss and gain by charge transfer also varies, demonstrating the level of charge transfer can be manipulated by adjusting the ratio of $\mathrm{MoO}_{3} \mathrm{NP}$ in the complex. To measure the modulation on energy levels from the charge transfer phenomenon, UPS measurements of the $\mathrm{NiO}: \mathrm{MoO}_{3}$-complex were carried out. The work function of the $\mathrm{NiO}: \mathrm{MoO}_{3}$-complex showed a considerable change of $1.87 \mathrm{eV}$ (from 4.47 to $6.34 \mathrm{eV}$ ), which is proportional to the $\mathrm{MoO}_{3}$ NPs fraction in the complex (Fig. 3e).
To compare the range of the work function change in the $\mathrm{NiO}: \mathrm{MoO}_{3}$-complex, a Mo-doped $\mathrm{NiO}$ system was also prepared with the same sol-gel fabrication conditions. The UPS measurement data (Supplementary Fig. 6) showed insignificant energy level shifts with increasing Mo concentrations in the complex, presumably due to the lack of charge transfer between the solid solution phase dopants and the $\mathrm{NiO}$ matrix, which is also supported by the smaller shift and change in the XPS spectra. This shows that the heterostructure charge transfer complex is a viable approach for modulating the energy level of the entire matrix through charge transfer by adjusting the amount of $\mathrm{MoO}_{3}$ $\mathrm{NPs}$ to the NiO: $\mathrm{MoO}_{3}$-complex.

Next, the electrical conductivity of the $\mathrm{NiO}: \mathrm{MoO}_{3}$-complex was evaluated by current-voltage measurements of the ITO/NiO:$\mathrm{MoO}_{3}$-complex/Au structure (Fig. 3f). Compared to that of pristine $\mathrm{NiO}$, the current density was improved by $79 \%$ for 15 at. $\% \mathrm{MoO}_{3} \mathrm{NPs}$ and continuously increased up to $140 \%$ with increasing $\mathrm{MoO}_{3} \mathrm{NP}$ ratio. It needs to be noted that the log-log plot of the current density-voltage $(J-V)$ shown in Fig. $3 \mathrm{f}$ follows the Ohmic conduction mechanism, which is dominated by electrical conductivity. The Hall effect measurement also provides a consistent trend of increasing conductivity for higher Mo fraction resulting from the enhanced carrier concentration (Supplementary Fig. 7). The increase in the conductivity is presumed to derive from further creation of charge carriers as a consequence of the charge transfer and $\mathrm{Ni}^{2+}$ vacancies formation 

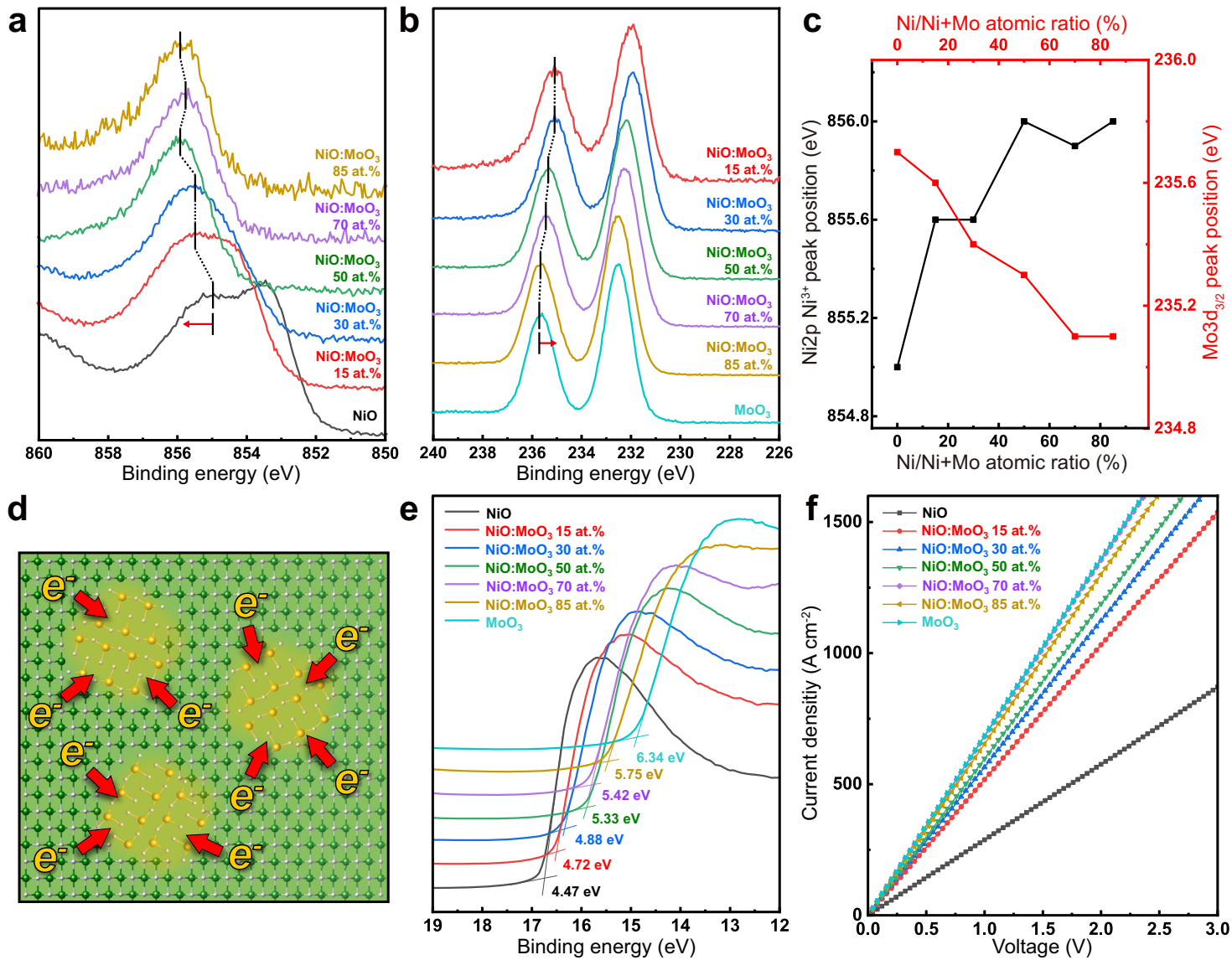

Fig. 3 X-ray photoelectron spectroscopy studies and electrical property characterization of NiO:MoO $\mathbf{3}_{\mathbf{3}}$-complex. XPS of a Ni $2 p$ and $\mathbf{b} \mathrm{Mo} 3 d$ narrow scan spectra of $\mathrm{NiO}: \mathrm{MoO}_{3}$-complex. Dashed lines refer to peak position shifts by the $\mathrm{MoO}_{3} \mathrm{NP}$ fraction. c Peak position shift of Ni $2 p \mathrm{Ni}{ }^{3+}$ and $\mathrm{Mo} 3 d_{3 / 2}$ with varying compositions of the $\mathrm{NiO}: \mathrm{MoO}_{3}$-complex. $\mathbf{d}$ Schematic of electron charge transfer between the $\mathrm{MoO}_{3} \mathrm{NP}$ and $\mathrm{NiO}$ interface. e Secondary cutoff spectra of ultraviolet photoelectron spectroscopy (UPS) and $\mathbf{f} I-V$ curves for $\mathrm{NiO}: \mathrm{MoO}_{3}$-complex.

during the fabrication process. Vacancy generation is supported by the deconvoluted $\mathrm{Ni} 2 p$ XPS spectra of the $\mathrm{NiO}: \mathrm{MoO}_{3}$ complex, which can be mainly divided into the $\mathrm{Ni}^{2+}$ peak of stoichiometric $\mathrm{NiO}$ and the $\mathrm{Ni}^{3}+$ peak of off-stoichiometric $\mathrm{NiO}$ containing $\mathrm{Ni}^{2+}$ vacancies, respectively (Supplementary Fig. 10). The $\mathrm{Ni}^{3+}$ peak ratio increases with an increasing fraction of $\mathrm{MoO}_{3} \mathrm{NPs}$ in the complex, which is relevant to increased carrier generation in the complex resulting in increased current density and the charge transport ability. The result of vacancy creation according to the $\mathrm{MoO}_{3}$ NPs ratio is also consistent with the structural analysis results from the HRTEM and XRD measurements shown in Fig. 2.

Performance improvement of $\mathrm{NiO}: \mathrm{MoO}_{3}-\mathrm{complex}$ based OLEDs. We applied the $\mathrm{NiO}: \mathrm{MoO}_{3}$-complex as an hole injection layer (HIL) in green phosphorescent OLED device to verify the ability of energy level modulation to improve device performance (Fig. 4). The current density-voltage-luminance $(J-V-L)$ characteristics were measured for various HILs such as pure $\mathrm{NiO}$, 10 at.\% to 30 at.\% of $\mathrm{NiO}: \mathrm{MoO}_{3}$ complex, and pure $\mathrm{MoO}_{3}$ with devices having the energy configuration shown in Fig. 4a. Overall, the current density and luminance decreased with an increasing $\mathrm{MoO}_{3} \mathrm{NP}$ fraction in the $\mathrm{NiO}: \mathrm{MoO}_{3}$-complex HIL (Fig. 4b). However, the pure $\mathrm{MoO}_{3} \mathrm{HIL}$ device showed slightly increased current density compared to the $\mathrm{NiO}: \mathrm{MoO}_{3} 30$ at.\% HIL, due to charge imbalance from the inadequate electron-blocking ability of $\mathrm{MoO}_{3}$. Compared to the current efficiencies of $\mathrm{NiO}\left(11.4 \mathrm{~cd} \mathrm{~A}^{-1}\right)$ and $\mathrm{MoO}_{3}$ devices $\left(5.6 \mathrm{~cd} \mathrm{~A}^{-1}\right)$ at $1000 \mathrm{~cd} \mathrm{~m}^{-2}$, the $\mathrm{NiO}: \mathrm{MoO}_{3^{-}}$ complex showed improved current efficiency, achieving $16.3 \mathrm{~cd}$ A $-1\left(\mathrm{MoO}_{3} 30\right.$ at. $\%$, at $\left.1000 \mathrm{~cd} \mathrm{~m}^{-2}\right)$, corresponding to $43 \%$ and $189 \%$ improved current efficiency compared to the $\mathrm{NiO}$ and $\mathrm{MoO}_{3}$ HILs. Each device showed consistent green electroluminescence spectra with peaks at $514 \mathrm{~nm}$ and CIE 1931 color coordinates of $(0.28,0.64)$, as shown in Fig. $4 \mathrm{~d}$ and Supplementary Fig. 11.

The notably improved OLED performance is attributed to the energy level modulation and optimization of the HILs. The $\mathrm{NiO}: \mathrm{MoO}_{3}$ complex achieved an excellent electron-hole charge balance by the well matched energy level configuration, which was difficult to achieve with the pure $\mathrm{NiO}$ and $\mathrm{MoO}_{3}$ HILs due to their inappropriate energy structure alignment. For a better understanding, Fig. 4e-g show the energy level diagrams of the OLED multilayer structure with $\mathrm{NiO}, \mathrm{NiO}: \mathrm{MoO}_{3}$-complex, and $\mathrm{MoO}_{3}$. For the $\mathrm{NiO}$ or $\mathrm{MoO}_{3} \mathrm{HIL}$, the injection energy level is located at levels that are too shallow or deep and thus result in unfavorable energy structure configurations, as shown in Fig. 4e and g. Therefore, either holes or electrons dominate the structures, resulting in a poor charge balance that leads to low device efficiency. The $\mathrm{NiO}: \mathrm{MoO}_{3}$-complex, on the other hand, is able to modulate the energy level to form a well-defined energy structure configuration and effective electron-hole balance, which enhances the overall performance of the device (Fig. 4f).

High capability and generality of NiO: $\mathrm{MoO}_{3}$-complex. Finally, the $\mathrm{NiO}: \mathrm{MoO}_{3}$-complex was applied to another optoelectronic configuration to investigate its generality as a HIL. Blue 

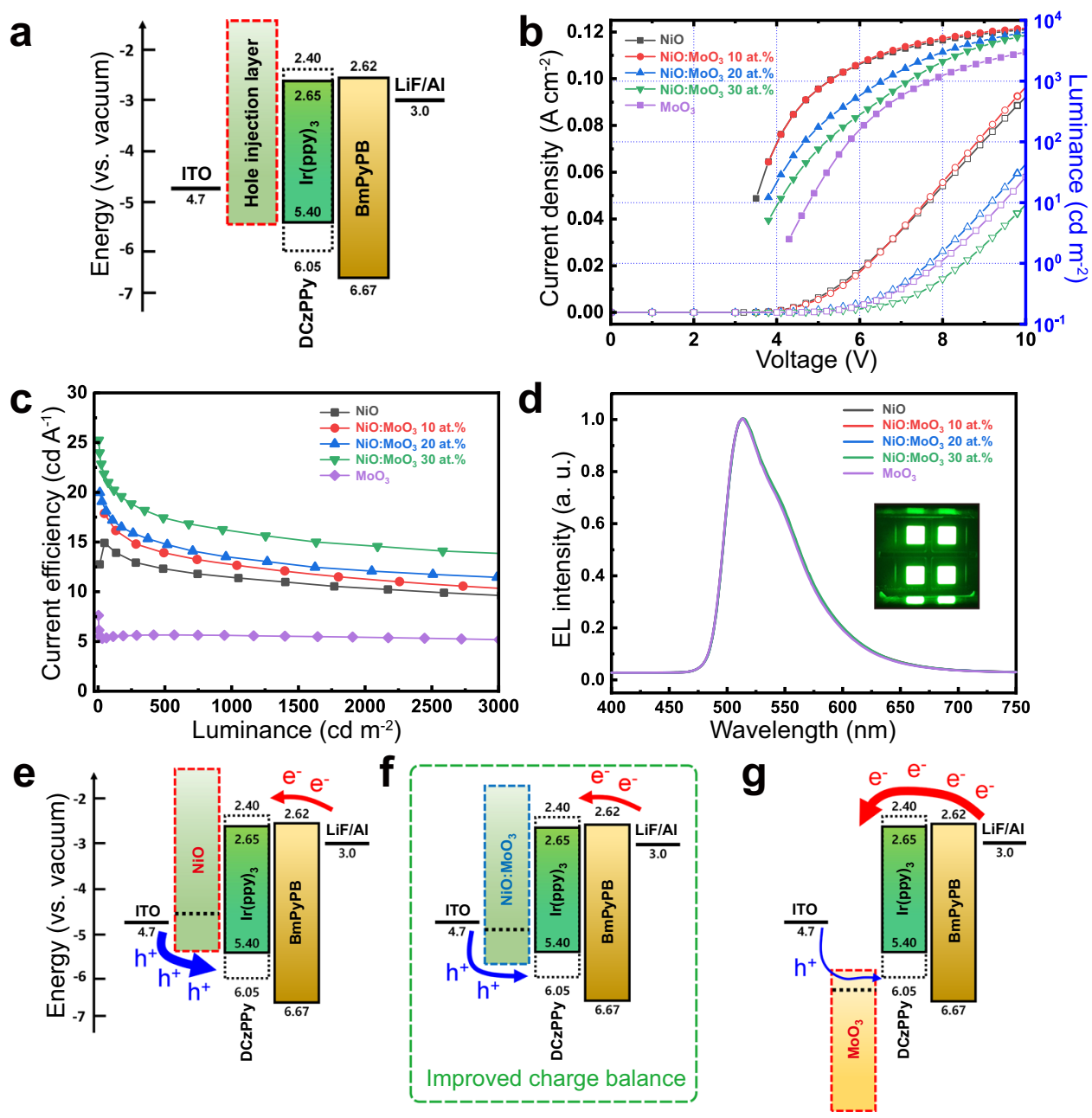

Fig. 4 Characterization of green phosphorescent OLEDs. a Energy level diagram of green phosphorescent OLED device. b Current density-voltage-luminance curves, c luminance-current efficiency curves, and $\mathbf{d}$ normalized electroluminescence for $\mathrm{NiO}, \mathrm{NiO} \mathrm{MoO}_{3}-\mathrm{complex}_{1}$ and $\mathrm{MoO}_{3}$ based OLEDs. The inset image in $\mathbf{d}$ shows an operating OLED device. Energy level and charge balance diagrams of multilayer structure configurations with e $\mathrm{NiO} \mathrm{HIL}$, f $\mathrm{NiO}: \mathrm{MoO}_{3}$-complex $\mathrm{HIL}$, and $\mathbf{g} \mathrm{MoO}_{3} \mathrm{HIL}$.

phosphorescent OLEDs were demonstrated, with a structure of ITO/HIL/TAPC/TCTA-DCzppy:Firpic/BmPyPB/LiF/Al (Fig. 5a). A TAPC HTL with high triplet energy and a TCTA-DCzppy:Firpic double EML were introduced with the purpose of inducing more efficient exciton formation and high device performance. We also evaluated HATCN besides $\mathrm{NiO}: \mathrm{MoO}_{3}$-complex as a HIL, which is widely used in various OLED structures, to clarify the generality of the $\mathrm{NiO}: \mathrm{MoO}_{3}$-complex. The $\mathrm{NiO}: \mathrm{MoO}_{3}$-complex based devices show a similar $J-V-L$ trend to those of the green phosphorescent OLED results above, revealing decreasing in current density and luminance with an increasing ratio of $\mathrm{MoO}_{3}$ NPs (Fig. 5b). However, the overall device performance and efficiency roll-off characteristics were notably improved when compared to green phosphorescent OLEDs. In terms of device performance, high current efficiency of $32.6 \mathrm{~cd} \mathrm{~A}^{-1}$ at $500 \mathrm{~cd} \mathrm{~m}^{-2}$ ( $17 \%$ of EQE) was achieved in the NiO: $\mathrm{MoO}_{3} 10$ at.\% device which is even higher than that of the HATCN device $\left(27 \mathrm{~cd} \mathrm{~A}^{-1}\right.$ at $500 \mathrm{~cd} \mathrm{~m}^{-2}, \sim 14 \%$ of EQE) (Fig. $5 \mathrm{c}$ and Supplementary Fig. 12). The detailed plots of each green and blue OLEDs were presented in Supplementary Fig. 13. As shown in Fig. 5d, consistent spectra of blue electroluminescence with $472 \mathrm{~nm}$ peak were observed for all devices. There were only slight spectrum shift in a broad range of $470-580 \mathrm{~nm}$. In addition, it is found that the device prepared with the charge transfer complex HIL was advantageous over the other approaches discussed earlier, from comparisons using a blue phosphorescent OLED system (Supplementary Fig. 14).

Consistent tendencies in $J-V-L$ characteristics across the different optoelectronic configurations indicate a definite operation of the $\mathrm{NiO}: \mathrm{MoO}_{3}$-complex with effective control of the electron-hole charge balance. Furthermore, we found that optimal device performances was obtained at a different $\mathrm{MoO}_{3}$ fraction for each device structure. This implies that optimum device structures do not always result from a single specific ratio of $\mathrm{NiO}: \mathrm{MoO}_{3}$-complex. Hence, the $\mathrm{NiO}: \mathrm{MoO}_{3}$-complex has huge potential for diverse optoelectronics such as a QLEDs (Supplementary Fig. 15) or PeLEDs, based on its general applicability due to the facile energy band tailoring ability.

\section{Discussion}

In summary, we introduced the formation of a heterostructure charge transfer complex as a unique approach to simultaneously modulate energy levels and enhance the electrical conductivity of a metal oxide system. A $\mathrm{NiO}$ matrix incorporating $\mathrm{MoO}_{3}$ nanoparticles of a few-nm size was demonstrated as an example of a heterostructure charge transfer system. In the $\mathrm{NiO}: \mathrm{MoO}_{3}$-complex, the energy level can be controlled by varying the $\mathrm{MoO}_{3} \mathrm{NPs}$ fraction in the complex because the total interfacial area defines the extent of charge transfer. XRD and HRTEM analyses revealed clearly distinct $\mathrm{NiO}$ and $\mathrm{MoO}_{3}$ phases, suggesting that their 

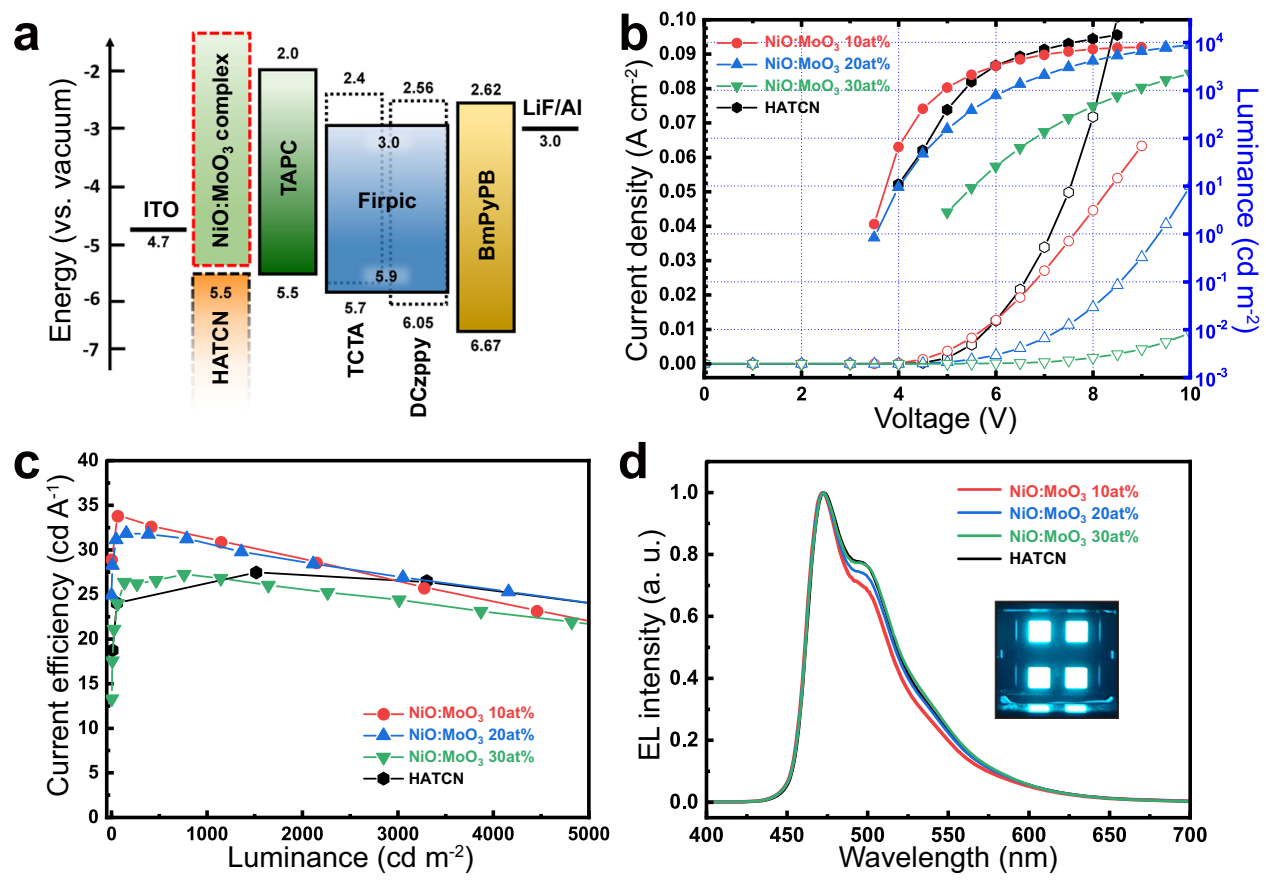

Fig. 5 Characterization of blue phosphorescent OLEDs. a Energy level diagram of blue phosphorescent OLED device. HATCN was performed as a comparison $\mathrm{HIL}$ with $\mathrm{NiO}: \mathrm{MoO}_{3}$-complex. b Current density-voltage-luminance curves, c luminance-current efficiency curves, $\mathbf{d}$ normalized electroluminescence for $\mathrm{NiO}: \mathrm{MoO}_{3}$-complex and HATCN based OLEDs. The inset image in $\mathbf{d}$ shows an operating OLED device.

individual energy structures are maintained at the nanoscale. Consequently, charge transfer between $\mathrm{NiO}$ and $\mathrm{MoO}_{3}$ was effectively induced throughout the whole heterostructures, extensively modulating their work function from 4.47 to $6.34 \mathrm{eV}$. The electrical conductivity was also enhanced by 2.4 times relative to that of pristine $\mathrm{NiO}$ through effective charge transfer and $\mathrm{Ni}^{2+}$ vacancy generation at the $\mathrm{NiO}-\mathrm{MoO}_{3} \mathrm{NP}$ interfaces. This energy band modulation ability leads to substantially improved current efficiency in green phosphorescent OLEDs with $\mathrm{NiO}: \mathrm{MoO}_{3}$ 30 at. $\%$ HIL, presenting $43 \%$ and $189 \%$ higher current efficiency than that of pristine $\mathrm{NiO}$ and $\mathrm{MoO}_{3}$ HILs due to well-defined energy band structure formation. Furthermore, blue phosphorescent OLEDs were also demonstrated to verify the high capability and generality of the $\mathrm{NiO}: \mathrm{MoO}_{3}-$ complex, showing $32.6 \mathrm{~cd} \mathrm{~A}^{-1}$ current efficiency and 17\% EQE with the $\mathrm{NiO}: \mathrm{MoO}_{3}$ 10 at.\% HIL, outperforming even the HATCN HIL device. Our strategy overcomes the limitations of conventional metal oxide enhancement methods by delocalizing charge transfer from the surface to the entire film, suggesting an alternative direction for applying metal oxide heterostructures to enhance the performance of multi-layered optoelectronic devices to a great extent.

\section{Methods}

Materials. Nickel(II) acetate tetrahydrate $\left(\left(\mathrm{CH}_{3} \mathrm{CO}_{2}\right) 2 \mathrm{Ni} \cdot 4 \mathrm{H}_{2} \mathrm{O}, 99 \%\right)$ was purchased from ACROS Organics. Bis(acac)dioxomolybdenum(VI) $\left(\left(\mathrm{CH}_{3} \mathrm{COCH}=\mathrm{C}(\mathrm{O}-) \mathrm{CH}_{3}\right)_{2} \mathrm{MoO}_{2}\right)$, ethanolamine $\left(\mathrm{NH}_{2} \mathrm{CH}_{2} \mathrm{CH}_{2} \mathrm{OH}, \geq 99.0 \%\right)$, 2-methoxyethanol $\left(\mathrm{CH}_{3} \mathrm{OCH}_{2} \mathrm{CH}_{2} \mathrm{OH}\right.$, anhydrous, $\left.99.8 \%\right)$, and hydrogen peroxide solution $\left(\mathrm{H}_{2} \mathrm{O}_{2}, 30 \mathrm{wt} \%\right)$ were supplied by Sigma-Aldrich. Ethanol $\left(\mathrm{C}_{2} \mathrm{H}_{5} \mathrm{OH}, \geq 99.9 \%\right)$ was prepared from Merck Millipore. All the organic chemicals were used as received without any further purification.

Synthesis of $\mathrm{NiO}$ precursor solution and $\mathrm{MoO}_{3}$ nanoparticles. To obtain a $0.1 \mathrm{M} \mathrm{NiO}$ precursor solution, $0.5 \mathrm{mmol}$ of nickel(II) acetate tetrahydrate, $30 \mu \mathrm{l}$ of ethanolamine, and $5 \mathrm{ml}$ of ethanol were blended and stored at $60^{\circ} \mathrm{C}$ for $12 \mathrm{~h}$ with stirring. $\mathrm{MoO}_{3}$ nanoparticles were obtained by a microwave-assisted synthesis method. First, $0.5 \mathrm{mmol}$ of molybdenum dioxide bis(acetylacetonate) $\left(\mathrm{C}_{10} \mathrm{H}_{16} \mathrm{MoO}_{6}\right)$ in $5 \mathrm{ml}$ of 2 -methoxyethanol was placed in a microwave reactor and heated by a microwave of $2.54 \mathrm{GHz}$ frequency for $60 \mathrm{~s}$. The synthesized $\mathrm{MoO}_{3}$ nanoparticles solution had a yellowish color. $\mathrm{MoO}_{3}$ nanoparticles have a monodisperse distribution with a $2.9 \mathrm{~nm}$ median (Supplementary Fig. 2). The NiO precursor and $\mathrm{MoO}_{3}$ nanoparticles solutions were then purified by using a PVDF ( $0.45 \mu \mathrm{m}$ pore size) filter.

Preparation of NiO: $\mathbf{M o O}_{3}$-complex films. First, $0.4 \mathrm{~mol}$ of hydrogen peroxide $\left(\mathrm{H}_{2} \mathrm{O}_{2}\right)$ was added to the prepared $0.1 \mathrm{M} \mathrm{MoO}_{3}$ nanoparticle solution. $\mathrm{H}_{2} \mathrm{O}_{2}$ acts as a stabilizer from the agglomeration of $\mathrm{MoO}_{3} \mathrm{NPs}$ in a blending solution of Ni precursor and $\mathrm{MoO}_{3}$ NPs (Supplementary Fig. 16, 17 and Supplementary Movie 1). A $\mathrm{MoO}_{3} \mathrm{NP}$ solution with $\mathrm{H}_{2} \mathrm{O}_{2}$ was then added to the NiO precursor solutions and blended with a Vortex mixer for $1 \mathrm{~min}$. The mixture solutions were purified with a PVDF $(0.45 \mu \mathrm{m}$ pore size $)$ filter and spin-coated on a cleaned ITO substrate at $3000 \mathrm{rpm}$ for $30 \mathrm{~s}$. The sample was then annealed at $300^{\circ} \mathrm{C}$ for $1 \mathrm{~h}$ in ambient conditions. The film thickness after spin-coating was $91.4-86.9 \mathrm{~nm}$, and the thickness decreased to $22.4-20.3 \mathrm{~nm}$ by the annealing process. Ellipsometry characterization combined with the Cauchy model was introduced to measure the film thickness containing solvent and moisture.

Characterization of NiO: $\mathbf{M o O}_{3}$-complex films. Scanning electron microscope (SEM) measurements were carried out to obtain images with a SU8230 (HITA$\mathrm{CHI}$ ). Structural characteristics of $\mathrm{NiO}, \mathrm{NiO}: \mathrm{MoO}_{3} \mathrm{NPs}$ complex, and $\mathrm{MoO}_{3} \mathrm{NPs}$ were investigated by transmission electron microscopy (HRTEM: JEM-2100F by JEOL, and HAADF-STEM: Talos F200X by FEI). Ultraviolet photoelectron spectroscopy (UPS) measurements were conducted with an Axis-Supra using a He I discharge lamp that has $21.2 \mathrm{eV}$ of photon energy under $9 \mathrm{~V}$ bias. The XRD patterns were obtained from an X-ray diffractometer (D/MAX-2500, RIGAKU) using $\mathrm{Cu}$ Ka X-ray $(\lambda=1.542 \AA)$. Atomic force microscopy (AFM) was implemented with a XE-70 Park Systems in non-contact mode with a SSS-NCHR cantilever. A Shimadzu UV-1800 spectrophotometer was employed to obtain the transmittance of the $\mathrm{NiO}: \mathrm{MoO}_{3}$-complex films. X-ray photoelectron spectroscopy (XPS) measurements were performed using a K-alpha (Thermo Fisher Scientific) with automated monochromatic X-ray source $\mathrm{Al}-\mathrm{Ka} 1486.7 \mathrm{eV}$ X-ray photons. Current-voltage curves were measured by source-measure unit (Keithley 238). Electrical resistivity (conductivity), Hall mobility, and carrier concentration were measured by Hall measurement with the van der Pauw method (HMS Model 8407 including high resistance configuration, Lake Shore Cryotronics Inc.).

Fabrication and measurements of OLEDs. Green phosphorescent OLEDs were fabricated with a multilayer structure as follows: indium-tin-oxide (ITO, $70 \mathrm{~nm}$ )/ HIL $(20 \mathrm{~nm}) / \mathrm{DCzPPy}: \operatorname{Ir}(\mathrm{ppy})_{3}(20 \mathrm{~nm}: 7 \%) / \mathrm{BmPyPB}(40 \mathrm{~nm}) / \mathrm{LiF}(1 \mathrm{~nm}) / \mathrm{Al}$ $(100 \mathrm{~nm})$. The patterned ITO glass substrate was cleaned by sonication in ethanol, acetone, and isopropanol for $20 \mathrm{~min}$, respectively. After UV-ozone treatment of the ITO substrate for $15 \mathrm{~min}$, the $\mathrm{NiO}: \mathrm{MoO}_{3}$-complex (HIL) was spin-coated on the substrates at $3000 \mathrm{rpm}$ for $30 \mathrm{~s}$ and then baked at $300^{\circ} \mathrm{C}$ for $1 \mathrm{~h}$ in ambient conditions. Dczppy:Ir(ppy) $)_{3}$ and the BmPyPB electron transport layer (ETL) were thermally deposited under ultrahigh vacuum $\left(P=10^{-8}\right.$ mbar $)$ with thickness of 20 
and $40 \mathrm{~nm}$, respectively (deposition rate $<1.0 \mathrm{~nm} \cdot \mathrm{s}^{-1}$ ). For the electron injection layer, $\mathrm{LiF}(1 \mathrm{~nm})$ was formed by thermal evaporation on the ETL. Finally, for the cathode layer, an aluminum layer of $100 \mathrm{~nm}$ thickness was thermally evaporated with a deposition rate of $0.3 \mathrm{~nm} \cdot \mathrm{s}^{-1}$. Blue phosphorescent OLEDs were fabricated with a multilayer structure of ITO/HIL/TAPC $(20 \mathrm{~nm}) /$ TCTA:Firpic $(10 \mathrm{~nm}: 7 \%) /$ Dczppy:Firpic (10 nm:10\%)/ BmPyPB (55 nm)/ LiF(1 nm)/ Al(100 nm). Processes except HIL deposition were sequentially thermally evaporated in the order of TAPC, TCTA:Firpic, Dczppy:Firpic, BmPyPB, LiF, and $\mathrm{Al}$ in ultrahigh vacuum $\left(P=10^{-8} \mathrm{mbar}\right)$. The current-voltage-luminance, current efficiency, and electroluminescence spectra were measured using a source-measure unit system (Keithley 238) and a goniometer-equipped spectroradiometer (Minolta CS-2000). EQE was calculated by using the assumption of a Lambertian distribution of the emission.

\section{Data availability}

The data that support the findings of this study are available from the corresponding author upon reasonable request.

Received: 27 December 2020; Accepted: 30 November 2021; Published online: 10 January 2022

\section{References}

1. Yu, X., Marks, T. J. \& Facchetti, A. Metal oxides for optoelectronic applications. Nat. Mater. 15, 383-396 (2016).

2. Liang, X. et al. Colloidal metal oxide nanocrystals as charge transporting layers for solution-processed light-emitting diodes and solar cells. Chem. Soc. Rev. 46, 1730-1759 (2017).

3. Wang, F., Tan, Z. A. \& Li, Y. Solution-processable metal oxides/chelates as electrode buffer layers for efficient and stable polymer solar cells. Energy Environ. Sci. 8, 1059-1091 (2015).

4. Fu, Q., Chen, J., Shi, C. \& Ma, D. Room-temperature sol-gel derived molybdenum oxide thin films for efficient and stable solution-processed organic light-emitting diodes. ACS Appl. Mater. Interfaces 5, 6024-6029 (2013).

5. Liu, S. et al. Nickel oxide hole injection/transport layers for efficient solutionprocessed organic light-emitting diodes. Chem. Mater. 26, 4528-4534 (2014).

6. Kim, K. et al. Conjugated polyelectrolyte hybridized $\mathrm{ZnO}$ nanoparticles as a cathode interfacial layer for efficient polymer light-emitting diodes. $A d v$. Funct. Mater. 25, 7450-7456 (2015).

7. Kirkwood, N., Singh, B. \& Mulvaney, P. Enhancing quantum dot LED efficiency by tuning electron mobility in the $\mathrm{ZnO}$ electron transport layer. Adv. Mater. Interfaces 3, 1600868 (2016)

8. Won, Y.-H. et al. Highly efficient and stable $\mathrm{InP} / \mathrm{ZnSe} / \mathrm{ZnS}$ quantum dot lightemitting diodes. Nature 575, 634-638 (2019).

9. Cho, H. et al. Highly efficient deep blue Cd-free quantum dot light-emitting diodes by a p-type doped emissive layer. Small 16, 2002109 (2020).

10. Kim, H.-M., Cho, S., Kim, J., Shin, H. \& Jang, J. Li and Mg co-doped zinc oxide electron transporting layer for highly efficient quantum dot lightemitting diodes. ACS Appl. Mater. Interfaces 10, 24028-24036 (2018).

11. Chan, I.-M., Hsu, T.-Y. \& Hong, F. C. Enhanced hole injections in organic light-emitting devices by depositing nickel oxide on indium tin oxide anode. Appl. Phys. Lett. 81, 1899-1901 (2002).

12. Bolink, H. J. et al. Inverted solution processable OLEDs using a metal oxide as an electron injection contact. Adv. Funct. Mater. 18, 145-150 (2008).

13. Caruge, J. M., Halpert, J. E., Wood, V., Bulović, V. \& Bawendi, M. G. Colloidal quantum-dot light-emitting diodes with metal-oxide charge transport layers. Nat. Photonics 2, 247-250 (2008).

14. Qian, L., Zheng, Y., Xue, J. \& Holloway, P. H. Stable and efficient quantumdot light-emitting diodes based on solution-processed multilayer structures. Nat. Photonics 5, 543-548 (2011).

15. Ma, H., Yip, H.-L., Huang, F. \& Jen, A. K.-Y. Interface engineering for organic electronics. Adv. Funct. Mater. 20, 1371-1388 (2010).

16. Ho, P. K. H. et al. Molecular-scale interface engineering for polymer lightemitting diodes. Nature 404, 481-484 (2000).

17. Ishii, H., Sugiyama, K., Ito, E. \& Seki, K. Energy level alignment and interfacial electronic structures at organic/metal and organic/organic interfaces. $A d v$. Mater. 11, 605-625 (1999).

18. Cao, F. et al. High-efficiency and stable quantum dot light-emitting diodes enabled by a solution-processed metal-doped nickel oxide hole injection interfacial layer. Adv. Funct. Mater. 27, 1704278 (2017).

19. Sun, Y. et al. High-performance quantum dot light-emitting diodes based on Al-doped $\mathrm{ZnO}$ nanoparticles electron transport layer. ACS Appl. Mater. Interfaces 10, 18902-18909 (2018).

20. Kim, M. et al. Conductivity enhancement of nickel oxide by copper cation codoping for hybrid organic-inorganic light-emitting diodes. ACS Photonics 5, 3389-3398 (2018).
21. Matsubara, K., Huang, S., Iwamoto, M. \& Pan, W. Enhanced conductivity and gating effect of p-type Li-doped $\mathrm{NiO}$ nanowires. Nanoscale 6, 688-692 (2014).

22. Xiang, D. et al. Surface transfer doping induced effective modulation on ambipolar characteristics of few-layer black phosphorus. Nat. Commun. 6, 6485 (2015)

23. Lin, J. D. et al. Electron-doping-enhanced trion formation in monolayer molybdenum disulfide functionalized with cesium carbonate. ACS Nano $\mathbf{8}$ 5323-5329 (2014).

24. Zhang, X., Shao, Z., Zhang, X., He, Y. \& Jie, J. Surface charge transfer doping of low-dimensional nanostructures toward high-performance nanodevices. Adv. Mater. 28, 10409-10442 (2016).

25. Luo, W. et al. Carrier modulation of ambipolar few-layer MoTe2 transistors by $\mathrm{MgO}$ surface charge transfer doping. Adv. Funct. Mater. 28, 1704539 (2018).

26. Liu, A., Zhu, H. \& Noh, Y.-Y. Molecule charge transfer doping for p-channel solution-processed copper oxide transistors. Adv. Funct. Mater. 30, 2002625 (2020).

27. Al-Ghamdi, A. A., Mahmoud, W. E., Yaghmour, S. J. \& Al-Marzouki, F. M. Structure and optical properties of nanocrystalline $\mathrm{NiO}$ thin film synthesized by sol-gel spin-coating method. J. Alloy. Compd. 486, 9-13 (2009).

28. Hellstrom, S. L. et al. Strong and stable doping of carbon nanotubes and graphene by MoOx for transparent electrodes. Nano. Lett. 12, 3574-3580 (2012).

29. Lin, J. et al. High-performance quantum-dot light-emitting diodes using $\mathrm{NiOx}$ hole-injection layers with a high and stable work function. Adv. Funct. Mater. 30, 1907265 (2020).

30. Jacob, K. T., Kale, G. M. \& Iyengar, G. N. K. Phase equilibria and thermodynamic properties in the system Ni-Mo-O. J. Mater. Sci. 22, 4274-4280 (1987)

31. Park, J. W., Kang, B. H. \& Kim, H. J. A review of low-temperature solutionprocessed metal oxide thin-film transistors for flexible electronics. Adv. Funct. Mater. 30, 1904632 (2020).

32. Bretos, I., Jiménez, R., Ricote, J. \& Calzada, M. L. Low-temperature crystallization of solution-derived metal oxide thin films assisted by chemical processes. Chem. Soc. Rev. 47, 291-308 (2018).

33. Lee, D., Kim, D., Lee, Y., Young \& Jeon, D. Solution processible MoOxincorporated graphene anode for efficient polymer light-emitting diodes. Nanotechnology 28, 235201 (2017).

34. Lee, Y.-J. et al. Low-temperature solution-processed molybdenum oxide nanoparticle hole transport layers for organic photovoltaic devices. Adv. Energy Mater. 2, 1193-1197 (2012).

35. Zhao, B. et al. Monolayer graphene/NiO nanosheets with two-dimension structure for supercapacitors. J. Mater. Chem. 21, 18792-18798 (2011).

36. Su, D., Ford, M. \& Wang, G. Mesoporous NiO crystals with dominantly exposed $\{110\}$ reactive facets for ultrafast lithium storage. Sci. Rep. 2, 924 (2012).

37. Chen, D. et al. Single-crystalline MoO3 nanoplates: topochemical synthesis and enhanced ethanol-sensing performance. J. Mater. Chem. 21, 9332-9342 (2011).

38. Kalantar-zadeh, K. et al. Synthesis of nanometre-thick MoO3 sheets. Nanoscale 2, 429-433 (2010).

39. Holder, C. F. \& Schaak, R. E. Tutorial on powder X-ray diffraction for characterizing nanoscale materials. ACS Nano 13, 7359-7365 (2019).

\section{Acknowledgements}

This research was supported by the Global Frontier Program through the Global Frontier Hybrid Interface Materials (GFHIM) (2013M3A6B1078874), ICT and Creative Materials Discovery Program (NRF-2016M3D1A1900035), and Nano-Material Technology Development Program (NRF-2021M3H4A3A01062963) of the National Research Foundation of Korea (NRF) funded by the Ministry of Science. This work was also supported by the ITECH R\&D Program of MOTIE/KEIT [Project no. 20012560, Development of material component equipment for inkjet printing in flexible QDOLED].

\section{Author contributions}

M.K. and Y.S.J conceived the project. M.K. and M.S.C. conducted most of the fabrication and the analysis experiments. M.K., B.-H.K. and C.W.J. contributed to OLED device fabrication and characterizations. Y.J.K. and H.C. contributed to XPS and UPS analysis. H.J. contributed to TEM analysis. M.K., E.N.C, and Y.S.J. wrote most of the manuscript All authors participated to the discussion of the results and writing of the manuscript.

\section{Competing interests}

The authors declare no competing interests. 


\section{Additional information}

Supplementary information The online version contains supplementary material available at https://doi.org/10.1038/s41467-021-27652-3.

Correspondence and requests for materials should be addressed to Eugene N. Cho or Yeon Sik Jung.

Peer review information Nature Communications thanks Jiansheng Jie and the other, anonymous, reviewer(s) for their contribution to the peer review of this work. Peer reviewer reports are available.

Reprints and permission information is available at http://www.nature.com/reprints

Publisher's note Springer Nature remains neutral with regard to jurisdictional claims in published maps and institutional affiliations. (c) (1) Open Access This article is licensed under a Creative Commons Attribution 4.0 International License, which permits use, sharing, adaptation, distribution and reproduction in any medium or format, as long as you give appropriate credit to the original author(s) and the source, provide a link to the Creative Commons license, and indicate if changes were made. The images or other third party material in this article are included in the article's Creative Commons license, unless indicated otherwise in a credit line to the material. If material is not included in the article's Creative Commons license and your intended use is not permitted by statutory regulation or exceeds the permitted use, you will need to obtain permission directly from the copyright holder. To view a copy of this license, visit http://creativecommons.org/ licenses/by/4.0/.

(C) The Author(s) 2022 